\title{
Targeting tumor-associated macrophages as a novel strategy against breast cancer
}

\author{
Yunping Luo, ${ }^{1,2}$ He Zhou, ${ }^{1}$ Jörg Krueger, ${ }^{1}$ Charles Kaplan, ${ }^{1}$ Sung-Hyung Lee, ${ }^{1}$ Carrie Dolman, ${ }^{1}$ \\ Dorothy Markowitz, ${ }^{1}$ Wenyuan Wu, ${ }^{1}$ Cheng Liu, ${ }^{1}$ Ralph A. Reisfeld, ${ }^{1}$ and Rong Xiang ${ }^{1}$
}

\begin{abstract}
1Department of Immunology, The Scripps Research Institute, La Jolla, California, USA. ${ }^{2}$ Key Laboratory of Laboratory Medical Diagnostics, Ministry of Education, Chongqing University of Medical Sciences, Chongqing, China.
\end{abstract}

\begin{abstract}
Tumor-associated macrophages (TAMs) are associated with tumor progression and metastasis. Here, we demonstrate for the first time that legumain, a member of the asparaginyl endopeptidase family functioning as a stress protein, overexpressed by TAMs, provides an ideal target molecule. In fact, a legumain-based DNA vaccine served as a tool to prove this point, as it induced a robust $\mathrm{CD8}^{+} \mathrm{T}$ cell response against $\mathrm{TAMs}$, which dramatically reduced their density in tumor tissues and resulted in a marked decrease in proangiogenic factors released by TAMs such as TGF- $\beta$, TNF- $\alpha$, MMP-9, and VEGF. This, in turn, led to a suppression of both tumor angiogenesis and tumor growth and metastasis. Importantly, the success of this strategy was demonstrated in murine models of metastatic breast, colon, and non-small cell lung cancers, where $75 \%$ of vaccinated mice survived lethal tumor cell challenges and $62 \%$ were completely free of metastases. In conclusion, decreasing the number of TAMs in the tumor stroma effectively altered the tumor microenvironment involved in tumor angiogenesis and progression to markedly suppress tumor growth and metastasis. Gaining better insights into the mechanisms required for an effective intervention in tumor growth and metastasis may ultimately lead to new therapeutic targets and better anticancer strategies.
\end{abstract}

\section{Introduction}

A novel antitumor strategy is immunization against molecules overexpressed by tumor-associated macrophages (TAMs) and thereby remodel the tumor microenvironment that attracts these macrophages and mediates their function $(1,2)$. TAMs consist primarily of a polarized M2 (F4/80+/CD206 $\left.6^{+}\right)$macrophage population with little cytotoxicity for tumor cells because of their limited production of NO and proinflammatory cytokines (3). TAMs also possess poor antigen-presenting capability and effectively suppress $T$ cell activation. In fact, these macrophages of M2 phenotype actually promote tumor cell proliferation and metastasis by secreting a wide range of growth and proangiogenic factors as well as metalloproteinases and by their involvement in signaling circuits that regulate the function of fibroblasts in the tumor stroma (4). In recent studies, anti-TAM effects induced by small molecule inhibitors contributed to tumor suppression $(5,6)$. For example, the antineoplastic agent Yondelis has a selective cytotoxic effect on TAMs, thereby significantly reducing their production of IL- 6 and CCL2, which, in turn, contribute to growth suppression of inflammation-associated human tumors (7). Another such example is provided by a biphosphonate compound, zoledronic acid, that suppresses MMP-9 secretion by TAMs, thereby inhibiting tumor metalloproteinase activity and diminishing the association of VEGF with its tyrosine kinase receptors on proliferating endothelial cells (8). In a different experimental model, the chemokine CCL5 was shown to be key in the recruitment of TAMs, and an antagonist of this chemokine reduced the tumor infiltrate and slowed tumor growth (9). Hence, although the therapeutic targeting of TAMs is still in its infancy, initial clinical results are encourag-

Nonstandard abbreviations used: TAM, tumor-associated macrophage. Conflict of interest: The authors have declared that no conflict of interest exists. Citation for this article: J. Clin. Invest. 116:2132-2141 (2006). doi:10.1172/JCI27648. ing, as they suggest that targeting TAMs may complement more conventional cancer treatment regimens.

Legumain is a novel evolutionary offshoot of the C13 family of cysteine proteases (10). It is well conserved in plants and mammals, including humans. It was first identified in plants as a processing enzyme of storage proteins during seed germination $(11,12)$ and was subsequently identified in parasites and then in mammals (13). Legumain is a robust acidic cysteine endopeptidase with remarkably restricted specificity, absolutely requiring an asparagine at the P1 site of its substrate sequence (13). The selection of legumain as a target for tumor therapy is based on the fact that the gene encoding this asparaginyl endopeptidase was found to be highly upregulated in many murine and human tumor tissues $(1,14,15)$ but absent or present only at very low levels in all normal tissues from which such tumors arise. Importantly, overexpression of legumain occurs under such stress conditions as tumor hypoxia, which leads to increased tumor progression, angiogenesis, and metastasis. In this regard, we recently discovered that legumain is heavily overexpressed by TAMs in murine breast tumor tissues by using gene expression profiling and immunohistochemistry. Importantly for our studies, TAMs have a particularly abundant expression in the tumor stroma (14) and express high levels of legumain in this tumor microenvironment. In contrast, classical macrophages of the M1 phenotype, which perform key immune-surveillance and antigen-presentation functions, do not express legumain. Consequently, targeting TAMs that overexpress legumain does not interfere with the biological functions of M1 macrophages, including cytotoxicity and antigen presentation (16-18).

Based on these findings, we hypothesized that targeting TAMs that overexpress legumain will reduce their density and thereby remodel the tumor microenvironment. This should lead to the downregulation of a wide variety of tumor growth factors, proangiogenic factors, and metalloproteinases released by these M2 
A

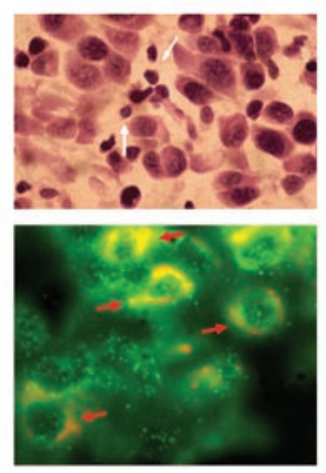

C

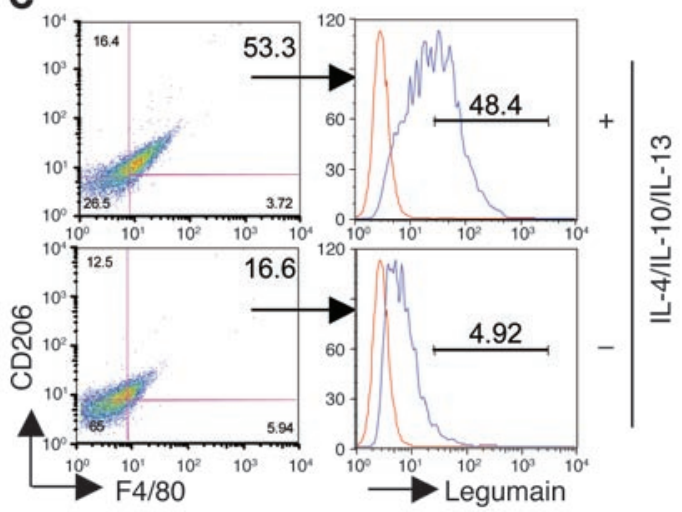

D

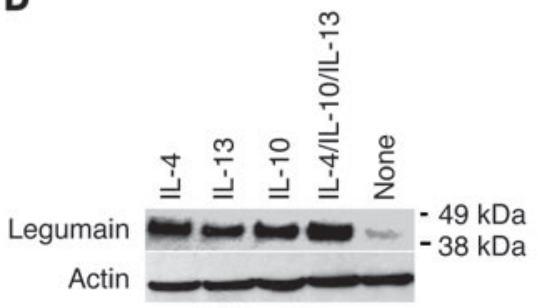

RAW cell line
B

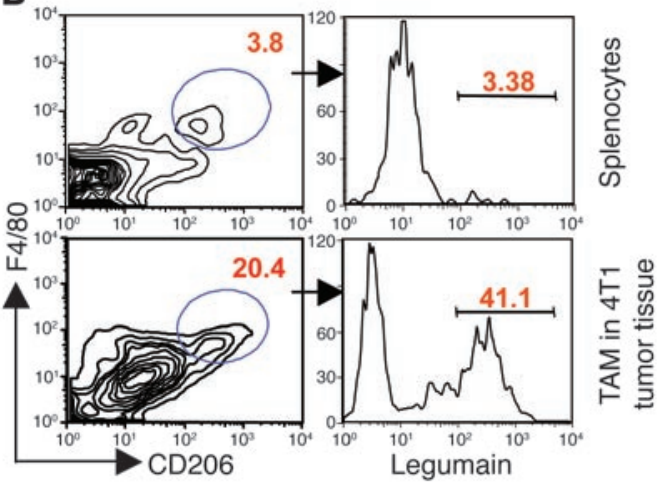

ing with anti-legumain $\mathrm{Ab}$ (Figure $1 \mathrm{~A}$, green) combined with anti-CD68 Ab (red). These data demonstrate that infiltrating TAMs are a disproportionally large cell subpopulation in 4T1 tumor tissue and that legumain is a potentially effective target for killing TAMs.

Induction of legumain expression on TAMs by Th2 cytokines. In order to determine whether legumain expression on TAMs was induced by such Th2 cytokines as IL-4, IL-10, and IL-13, we cocultured a murine macrophage cell line, RAW, with these cytokines. This resulted in a significant increase in $\mathrm{F} 4 / 80^{+} / \mathrm{CD}^{2} 06^{+}$expression by these RAW cells, concurrent with an upregulation of legumain (Figure 1C). These results were confirmed by Western blotting (Figure 1D). However, we found no evidence for legumain expression by tumor cell lines when cultured with these same cytokines (data not shown). These findings suggest that Th2 cytokines such as IL-4, IL-10, and IL-13 are released by tumor and other tumor stromal cells and accumulate in the tumor microenvironment, where they could potentially induce the proliferation and transformation from M1 macrophages to a population with an M2 phenotype that overexpresses legumain.

macrophages and thereby decisively suppress angiogenesis of tumors as well as their growth and metastasis. To test our hypothesis, a legumain-based DNA vaccine served as a tool to eliminate TAMs in murine models of colon, breast, and lung tumor metastases.

\section{Results}

Legumain serves as a target to kill TAMs overexpressed during tumor progression. It is well known that TAMs play a key role in tumor progression and metastasis (5). Therefore, targeting of these M2 macrophages represents a novel antitumor strategy. We initially identified legumain as a significant marker molecule of TAMs, since it was highly overexpressed on these cells in the tumor microenvironment and stroma. To this end, we isolated TAMs from murine 4T1 breast tumor tissue and demonstrated by flow cytometry that legumain was highly overexpressed on CD206 and F4/80 double-positive M2 macrophages, especially when compared with normal M1 macrophages in the spleen (Figure 1B). This result was also confirmed by immunohistochemical analyses, indicating that TAMs could be visualized by $\mathrm{H} \& \mathrm{E}$ staining, and legumain overexpression was further indicated by double stain-

Targeting of TAMs suppresses tumor progression. Growth and metastases of tumors are highly coordinated with the presence of TAMs, and therefore targeting of this macrophage subpopulation leads to suppression of tumor growth and metastases. To test this hypothesis, we generated an expression vector for a DNA vaccine encoding legumain. Figure $2 \mathrm{~A}$ schematically depicts the vector construction map based on the $\mathrm{pCMV/myc/cyto} \mathrm{vector}$ backbone. The gene encoding legumain was fused to the $\mathrm{C}$ terminus of mutant polyubiquitin (pLegumain) to improve antigen processing in the proteasome (19), and the entire fragment was then inserted between the PstI and NotI restriction sites, while protein expression was demonstrated by Western blotting. We further tested our hypothesis that reducing the number of TAMs by our legumain-based DNA vaccine can effectively inhibit spontaneous $4 \mathrm{~T} 1$ breast cancer metastases or experimental metastases of either D121 non-small cell lung or CT26 colon carcinomas. Thus, in a prophylactic setting, C57BL/6J mice were immunized 3 times with either PBS, empty vector, or pLegumain carried by attenuated Salmonella typhimurium. One week after the last immunization, these mice were challenged i.v. with $2 \times 10^{5}$ D 121 
A
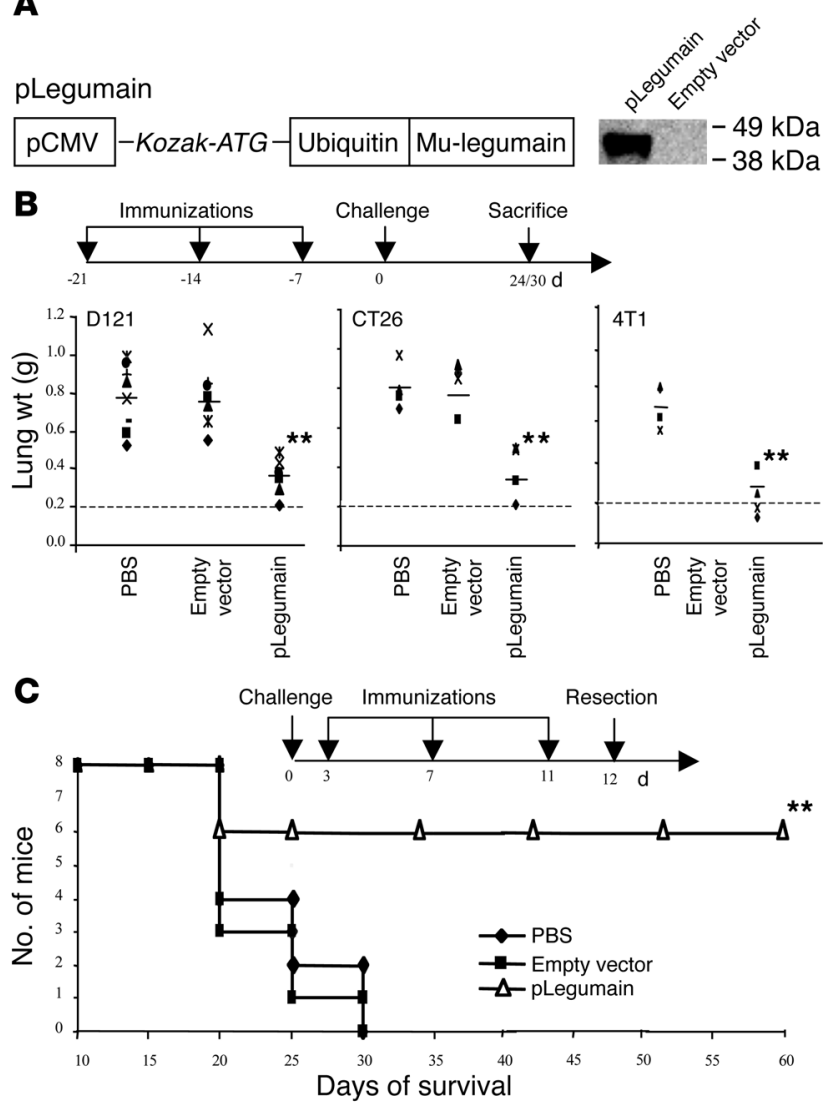

non-small lung tumor cells, and 24 days thereafter experimental lung metastases were measured and analyzed. In the 2 control groups, the average lung weight was significantly greater than that of the vaccination group (Figure 2B). Similar results were obtained in the CT26 colon tumor model and 4T1 breast cancer model in syngeneic BALB/c mice (Figure 2B).

In a more demanding therapeutic setting, $B A L B / c$ mice were first challenged with $4 \mathrm{~T} 1$ breast cancer cells and then immunized 3 times with the legumain-based DNA vaccine or an empty control vector. Twelve days after challenge with $4 \mathrm{~T} 1$ tumor cells, the primary tumor was surgically excised, and the resulting lifespan curve indicated that $75 \%(6 / 8)$ of the mice immunized with pLegumain survived for 3 months. In contrast, mice in the control groups all died within 1 month (Figure 2C). These data indicate that the legumain-based DNA vaccine effectively suppresses tumor cell growth and metastases in mouse models of 4T1 breast cancer, D121 non-small cell lung cancer, and CT26 colon carcinoma. Combined with surgery, this vaccine could indeed significantly extend the life span of mice by inhibiting tumor cell metastases in these very challenging therapeutic mouse tumor models.

Targeting legumain induces a specific $C D 8^{+} \mathrm{CTL}$ response, decreasing TAM populations in the tumor stroma. Immunization against legumain induced a specific $T$ cell response against TAMs that highly express this asparaginyl endopeptidase. This was demonstrated by a ${ }^{51} \mathrm{Cr}$ release assay, in which splenocytes isolated from mice successfully immunized with this vaccine were effective in killing RAW macrophages, which expressed high levels of legumain after culture with cytokines IL-4, IL-10, and IL-13; however, these

\section{Figure 2}

Targeting of legumain-expressing cells results in suppression of tumor progression. (A) Schematic of DNA vaccines constructed with the $\mathrm{pCMV/myc/cyto} \mathrm{vector} \mathrm{backbone} \mathrm{where} \mathrm{the} \mathrm{legumain} \mathrm{gene} \mathrm{was}$ fused to the $C$ terminal of mutant polyubiquitin. The entire fragment was inserted, and protein expression was demonstrated by Western blotting. Mu-legumain, murine legumain. (B) Prophylactic model: The vaccination schedule was designed for 3 immunizations at 1 -week intervals followed by an i.v. challenge with $2 \times 10^{5} \mathrm{D} 121$ non-small cell lung cancer cells and $5 \times 10^{4}$ CT26 colon cancer cells and mammary fat pad injection with $7 \times 10^{3} 4 \mathrm{~T} 1$ breast cancer cells. Lung weights were determined 24 days (D121 or CT26) or 30 days (4T1) after tumor cell challenge and analyzed in each group. Differences between the 2 control groups (PBS and/or empty vector) and the treatment group were statistically significant; ${ }^{* \star} P<0.005$. Pre-challenge lung weight, 0.2 g. (C) Therapeutic model: Groups of BALB/c mice $(n=8)$ were initially injected in the mammary fat pad with $7 \times 10^{3} 4 \mathrm{~T} 1$ breast cancer cells and thereafter vaccinated 3 times on days 3,7 , and 11 with PBS, empty vector, or the pLegumain vaccine, respectively, and primary tumors excised on day 12. Survival plots represent results for 8 mice in each of the treatment and control groups. The difference between the empty vector control group and the treatment group was statistically significant; ${ }^{* *} P<0.005$.

same splenocytes failed to induce cytotoxic killing of cells that lacked legumain expression (Figure 3A), indicating the specificity of this $\mathrm{T}$ cell response against legumain. Additionally, the same result was obtained using legumain-transfected cells as target cells in ${ }^{51} \mathrm{Cr}$ release assays (Supplemental Figure 1; available online with this article; doi:10.1172/JCI27648DS1). Furthermore, the results depicted in Figure 3B demonstrate a dramatic decrease in the $\mathrm{F} 4 / 80^{+} / \mathrm{CD}^{206} 6^{+}$macrophage population after legumainbased DNA vaccine treatment. These data were also confirmed by immunohistochemical staining, as shown in Figure 3C.

$M H C$ class I-restricted CD $8^{+} C T L$ s are specifically active against TAMs. In gaining some insight into the immune mechanisms involved in the cytotoxic activity against TAMs, we found that DCs in Peyer's patches of successfully immunized mice were activated 3 days after vaccination with pLegumain, as indicated by the upregulated DC activation markers CD40, CD80, and MHC class I (Figure 4A). Furthermore, $\mathrm{CD}^{+} \mathrm{T}$ cell activation was found to be specific for legumain, as indicated by double staining for IFN- $\gamma$ and CD8 on splenocytes obtained from successfully vaccinated mice (Figure 4B) and by the specific release of IFN- $\gamma$ by activated $\mathrm{T}$ cells stimulated with legumain-positive cells (Figure 4C). In addition, in vivo immune depletion of $\mathrm{CD}^{+}$or $\mathrm{CD}^{+} \mathrm{T}$ cells revealed that only $\mathrm{CD} 8^{+} \mathrm{T}$ cells play a major role in the specific cytotoxic killing of TAMs, since only their depletion completely abrogated this killing effect. This specific cytotoxicity was MHC class I antigen restricted, as killing was specifically inhibited by anti-H-2 $\mathrm{D}^{\mathrm{d}} / \mathrm{H}-2 \mathrm{~K}^{\mathrm{d}}$ antibodies (Figure $4 \mathrm{D}$ ). Taken together, our results suggest that the legumain-based DNA vaccine first activated DCs in Peyer's patches, after which these cells presented legumain peptides through the MHC class I antigen pathway to the TCR on activated $\mathrm{CD} 8^{+} \mathrm{T}$ cells, resulting in a specific cytotoxic $\mathrm{CD} 8^{+} \mathrm{T}$ cell response abrogating TAMs.

Abrogation of TAMs in the tumor stroma reduces the release of tumor growth factors and proangiogenic factors as well as inbibiting tumor cell migration and metastases. TAMs can influence tumor metastasis in several ways, as they secrete a wide variety of tumor growth factors, proangiogenic factors, and tumor-associated enzymes that 
A

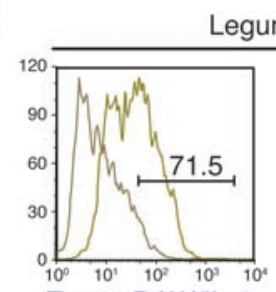

Target RAW/IL-4, $-10,-13$

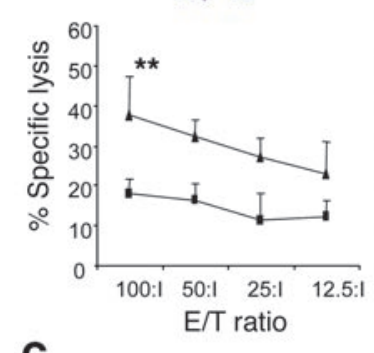

C
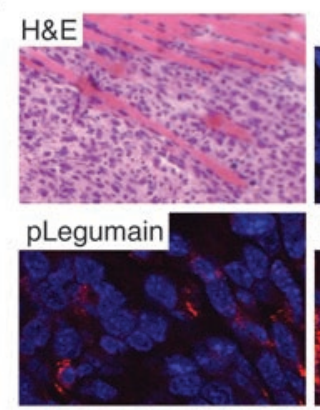

gumain expression

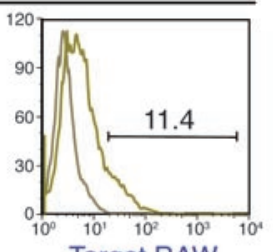

Target RAW
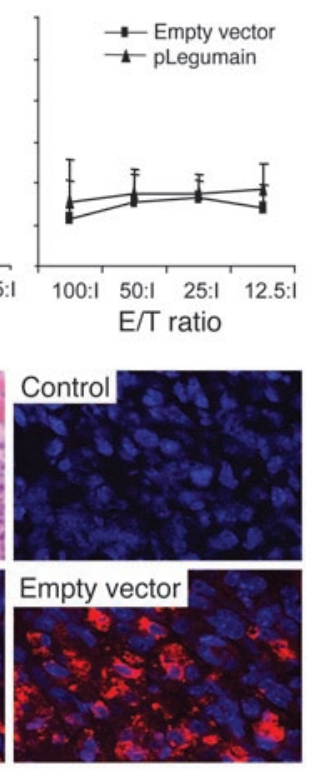

B
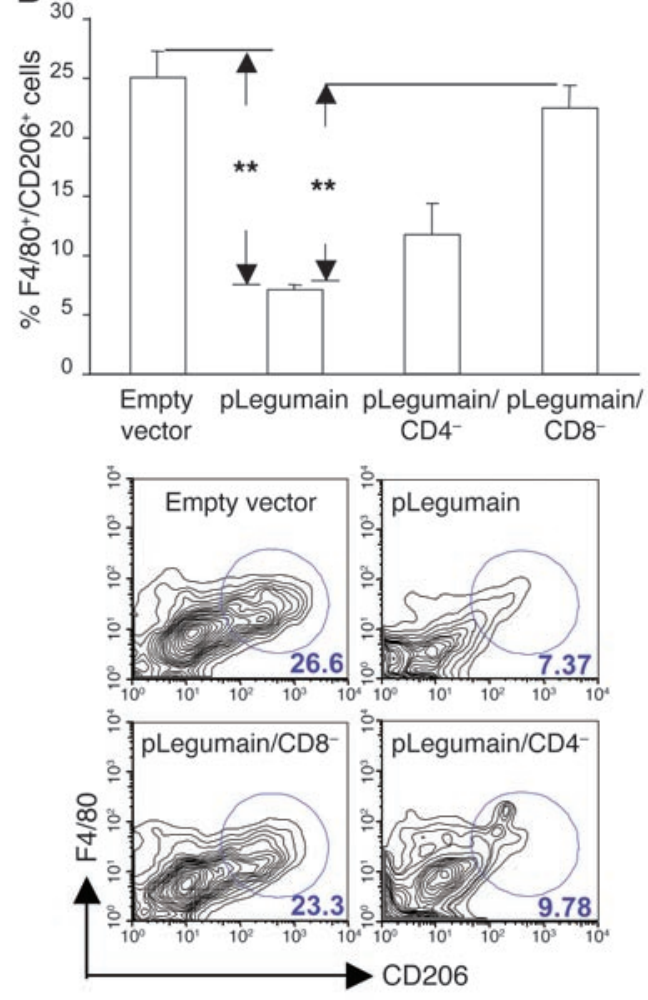

Figure 3

TAM population in the tumor stroma is decreased by CD8+-specific CTLs induced by the legumain-based DNA vaccine. (A) RAW macrophage cells, which highly express legumain after culturing with $10 \mathrm{ng} / \mathrm{ml} \mathrm{IL-4,} \mathrm{IL-10,} \mathrm{and} \mathrm{IL-13,} \mathrm{served} \mathrm{as} \mathrm{target} \mathrm{cells} \mathrm{in} \mathrm{a} \mathrm{4-hour}{ }^{51} \mathrm{Cr}$ release assay. Splenocytes isolated from mice immunized with the pLegumain vaccine were shown to effectively kill RAW cells treated with these cytokines in vitro at different effector-to-target $(E / T)$ cell ratios but failed to induce cytotoxic killing of unstimulated RAW cells lacking legumain expression. ${ }^{* \star} P<0.005$ compared with control groups. (B) The percentage of TAM populations with specific macrophage markers (CD206 and F4/80) in tumor tissue with or without vaccination was detected by flow cytometry. The percentage of TAM populations among tumor tissue cells isolated from mice treated with our DNA vaccine was shown to be reduced; however, there was no decrease in TAM populations isolated from mice treated with either empty vector or pLegumain following CD8 ${ }^{+} T$ cell depletion ( $\left.{ }^{\star \star} P<0.005\right)$. (C) The results of flow cytometry were confirmed by immunohistochemical staining evaluated by confocal microscopy. The population of TAMs in the tumor stroma was dramatically decreased after vaccination. 4T1 cancer cells are shown in blue and TAMs in red. Magnification, $\times 50$ (H\&E) and ×350 (control, empty vector, and pLegumain).

stimulate tumor angiogenesis and tumor growth and metastasis. In an effort to assess whether the elimination of TAMs actually reduced the release of some of these factors, serum and tumor tissue cells were collected from vaccinated mice and from suitable control animals. Freshly isolated tumor cells were cultured and their supernatants collected at 24 and 48 hours, respectively. An ELISA, performed to quantify TNF- $\alpha$, VEGF, and TGF- $\beta$, indicated a significant reduction in TNF- $\alpha$ and VEGF in both tumor cell supernatants and mouse serum; however, TGF- $\beta$ was significantly reduced only in serum but not in cell supernatants (Figure 5A). Immunohistological staining confirmed a decrease in the expression of these factors in tumor tissue (Figure 5B). In addition, a significantly decreased tumor cell migration was found when treatment and control groups were compared (Figure 5C) in a migration and invasion assay, which indicated that these characteristics of tumor cells changed after the vaccineinduced remodeling of the tumor microenvironment caused by the reduction in TAMs. The ability to form tumor metas- tases was confirmed in an in vivo experiment as the metastasis scores and lung weights - measured 24 days after primary tumor excision in a therapeutic setting, as described in Methods - decreased significantly when compared with the 2 control groups (Figure 5D).

Elimination of TAMs in the tumor stroma results in reduction of tumor angiogenesis. Importantly, there also was a marked antiangiogenic effect after elimination of TAMs in the tumor stroma, particularly since these M2 macrophages produced a wide range of proangiogenic factors. This was established by Matrigel assays that detected new blood vessel growth in vivo, an effect that could be quantified by staining the endothelium with FITC-labeled isolectin B4. These results clearly show that vessel growth was significantly reduced after vaccination with pLegumain (Figure 6B). It was also clearly indicated that much more blood vessels grew in Matrigel plugs in mice immunized with empty vector after evaluation by digital imaging and with Masson's trichrome staining (Figure 6A). Furthermore, an immunochemical histology assay 
A

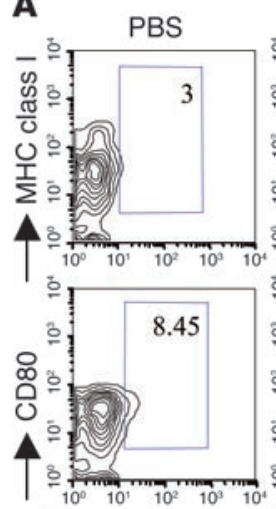

Empty vector
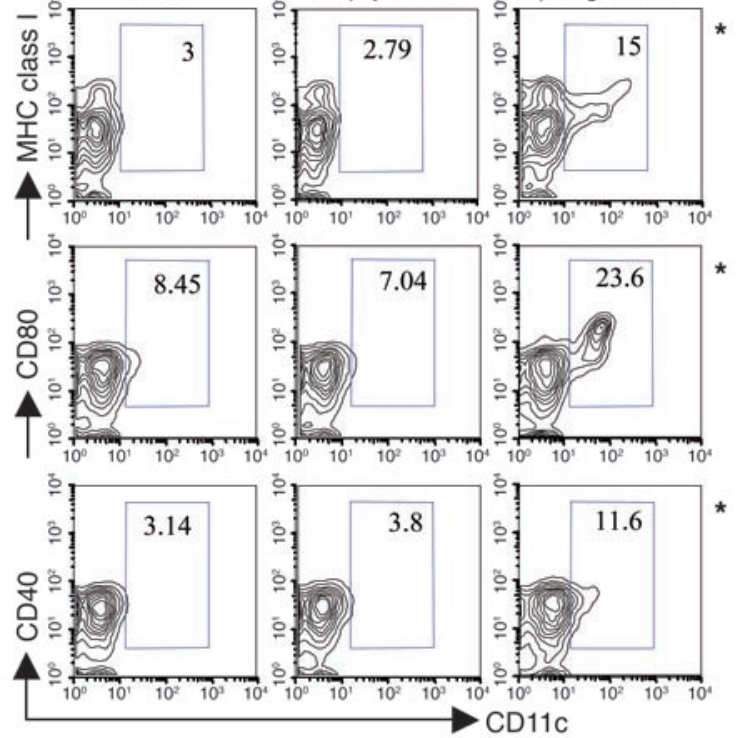

D
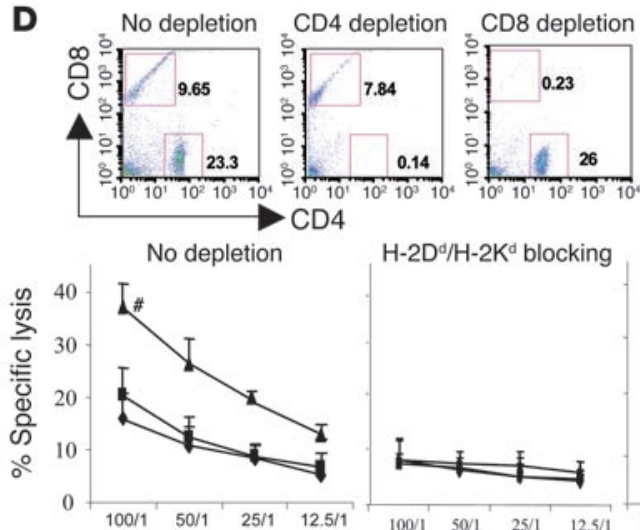

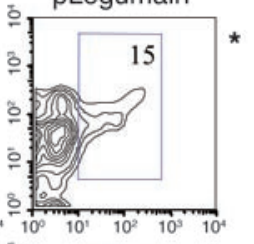

pLegumain
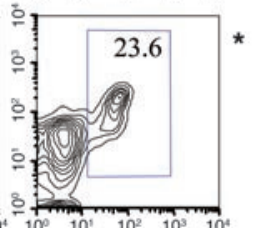

23

26
CD4 depletion

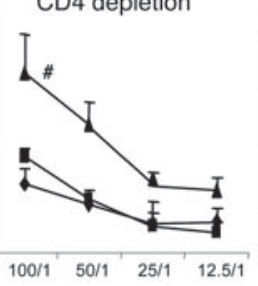

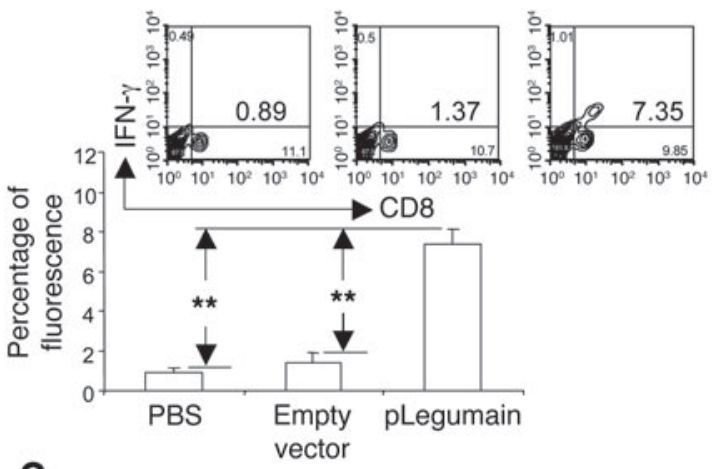

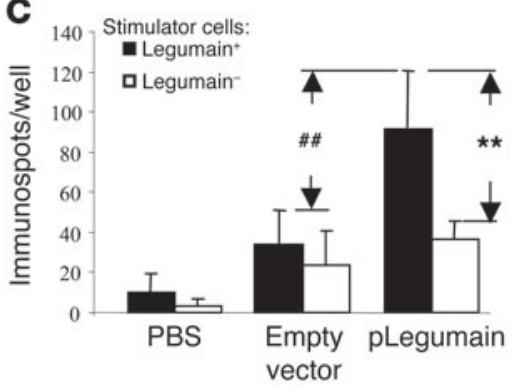

Figure 4

MHC class I antigen-restricted specific CD8 ${ }^{+} \mathrm{T}$ cell response against legumain-expressing cells. (A) DNA vaccination enhances expression of costimulatory molecules by DCs. Lymphocytes from Peyer's patches obtained 3 days after vaccination were stained with FITC-labeled anti$\mathrm{CD} 11 \mathrm{cAb}$ in combination with PE-conjugated anti-CD80, anti-MHC class I, or anti-CD40 Abs. ${ }^{*} P<0.05$ compared with control groups. (B) Intracytoplasmic IFN- $\gamma$ release of CD8 ${ }^{+} \mathrm{T}$ cells was measured by FACS analysis. ${ }^{* *} P<0.005$ compared with control groups. (C) Production of specific IFN- $\gamma$ was verified at the single-cell level by ELISPOT. This is indicated for lymphocytes from immunized mice restimulated with either legumain+ 4 T1 tumor tissue cells or legumain- 4T1 cells, as indicated by the number of immunospots formed per well. ${ }^{* *} P<0.005$ compared with treatment group without stimulation; \#\# $P<0.005$ compared with control groups. (D) Splenocytes isolated from treated mice were effective in killing TAMs according to a ${ }^{51} \mathrm{Cr}$ release assay ( $P<0.01$ compared with control groups). Inhibition experiments with Abs against $\mathrm{H}-2 \mathrm{~K}^{\mathrm{d}} / \mathrm{H}-2 \mathrm{D}^{\mathrm{d}} \mathrm{MHC}$ class I antigens showed that $\mathrm{T}$ cell-mediated tumor cell lysis was $\mathrm{MHC}$ class I antigen restricted. Furthermore, in vivo depletions of $\mathrm{CD}^{+}{ }^{+}$or $\mathrm{CD}^{+} \mathrm{T}^{+}$cells indicated that lymphocytes isolated from vaccinated mice, which were thereafter depleted of CD8 ${ }^{+} \mathrm{T}$ cells, failed to induce cytotoxic killing of target cells. However, depletion of $\mathrm{CD}^{+} \mathrm{T}$ cells did not abrogate cytotoxic killing of these same target cells. ${ }^{\#} P<0.01$ compared with PBS or empty vector group.

was performed to assess the type of cells that actually migrated into the Matrigel plugs. The confocal images taken indicated that endothelial cells expressing CD $31^{+}$or macrophages expressing $\mathrm{CD} 8^{+}$grew or migrated into Matrigel plugs to a considerably greater extent in the empty vector control group than in the vaccine treatment group (Figure 6C).

\section{Discussion}

This study establishes the new paradigm whereby a reduction in the density of TAMs in the tumor stroma decreases the release of factors potentiating tumor growth and angiogenesis. This, in turn, remodels the tumor microenvironment so as to markedly suppress tumor cell proliferation, vascularization, and metastasis. However, targeting TAMs in the tumor stroma raises the concern that their abrogation could interfere with the normal immunological functions of these important components of the innate immune system. We addressed this question in view of the fact that circulating monocytes are versatile precursors with the ability to differentiate into the various forms of specialized macrophages (3). In fact, the cytokine milieu profoundly affects the differentiation and function of tissue macrophages, and their functional polarization has been defined $(3,20,21)$. Thus, macrophages 
A

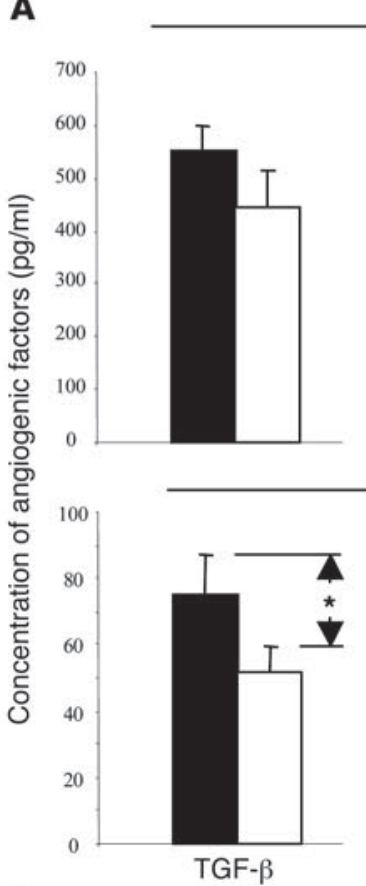

In supernatant

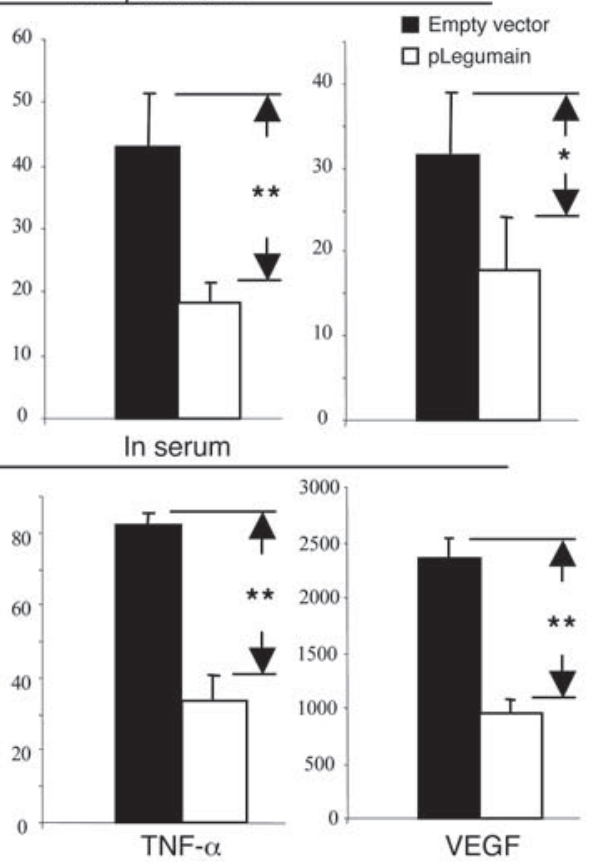

C

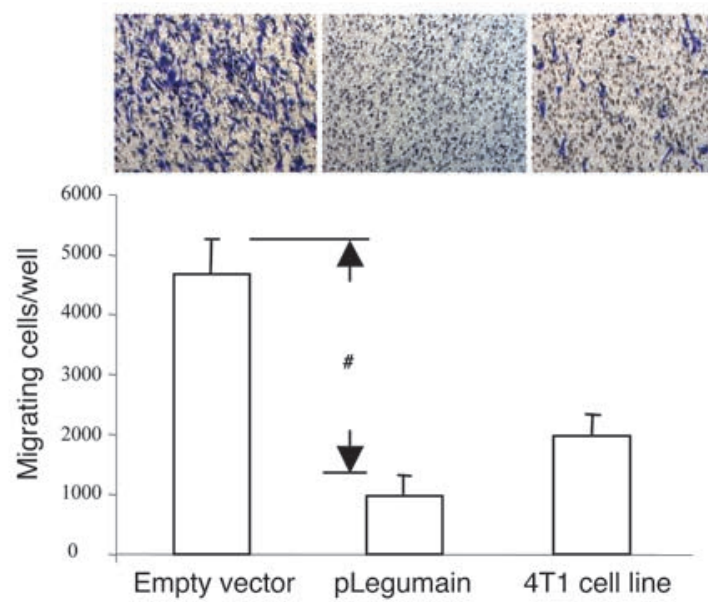

B

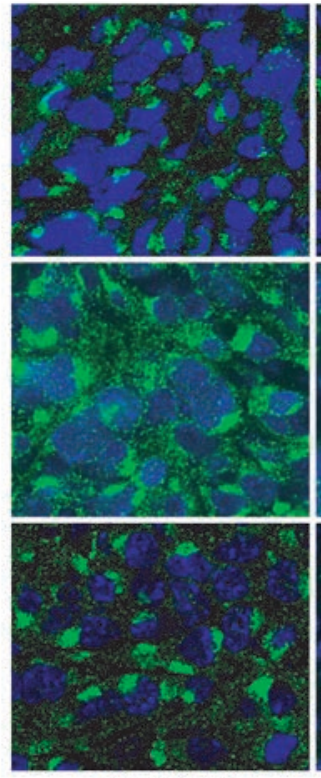

Empty vector
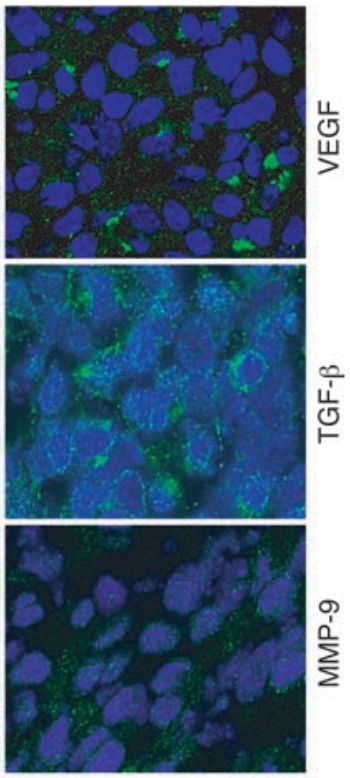

pLegumain

D

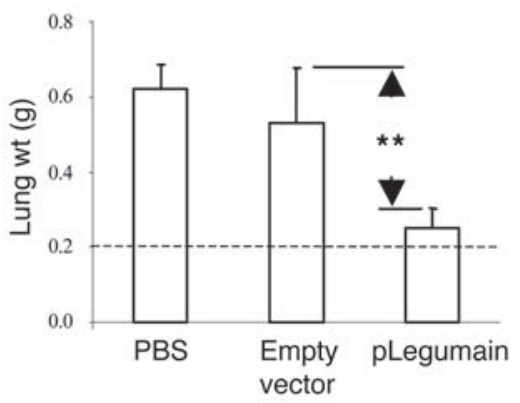

Figure 5

Abrogation of TAMs results in decreases of growth factor release, tumor cell migration, and metastases. (A) The vaccine decreased the release of growth factors by TAMs. 4T1 breast tumor tissue and mouse serum were harvested 12 days after vaccinations and tumor cell challenge. After 24 or 48 hours culturing, the supernatants of tumor tissue cells were harvested, and the concentrations of TGF- $\beta$, TNF- $\alpha$, and VEGF in serum or supernatants measured by ELISA. There were significant differences between the treatment and control groups. ${ }^{\star} P<0.01 ;{ }^{\star \star} P<0.005$. (B) Immunohistochemical staining was performed to determine expression of these growth factors in the tumor microenvironment. In the vaccine treatment groups, VEGF, TGF- $\beta$, and MMP-9 release was decreased after a reduction in TAMs, compared with the empty vector groups. The growth factors are shown in green and 4T1 breast cancer cells in blue. (C) A Transwell migration assay was performed to determine tumor cell migration after vaccination. The number of migrating cells isolated from $4 \mathrm{~T} 1$ tumor tissue was markedly reduced after vaccination. ${ }^{\#} P<0.001$ compared with the empty vector group. (D) In vivo experiments were performed to determine the ability of mice to form 4T1 tumor metastases. The mice were treated with the vaccine within the therapeutic setting as described above. Tumor metastasis scores and lung weights were measured 25 days after primary tumor excision. The metastasis scores are expressed as the percentage of lung surface covered by fused metastatic foci; 0: none; 1: $<5 \%$; 2: 5- 50\%; 3: >50\%. Scores for $n=8$ mice/group were: PBS, 3, 3, 3, 3, 3, 3, 2, 2; empty vector, 3, 3, 3, 3, 3, $3,3,2$; pLegumain, 2, 2, $10,0,0,0,0$. Differences in lung weights between the group of mice treated with vaccine and all control groups were statistically significant $\left({ }^{* *} P<0.005\right)$. Magnification, $\times 350(\mathbf{B}), \times 50(\mathbf{C})$.

activated by bacterial products and Th1 cytokines are regarded as being of the M1 phenotype, i.e., classically activated macrophages with high bactericidal activity and cytotoxic function against tumor cells. However, macrophages activated by such Th2 cytokines as IL-4 and IL-13 or immunosuppressors such as vitamin D3 and IL-10 are classified as macrophages with an M2 phenotype: low cytotoxic functions but high tissue-remodeling activity. Whereas M1 cells have immunostimulatory properties and 
A
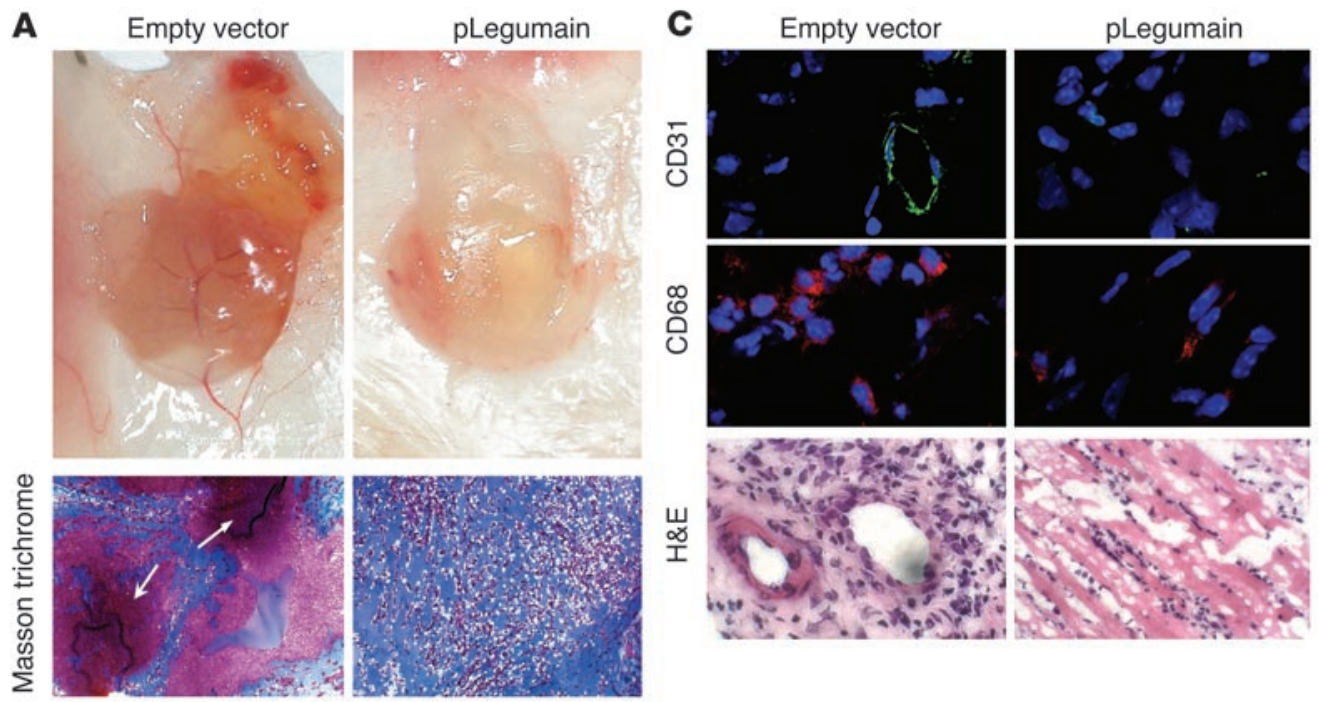

B

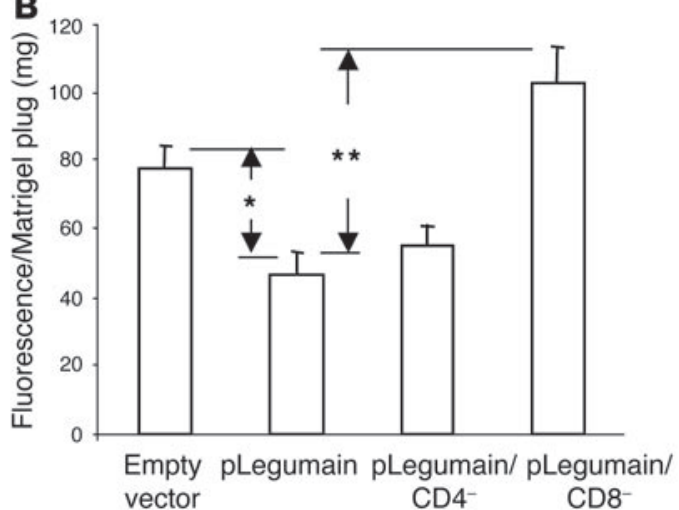

\section{Figure 6}

Elimination of TAMs results in a reduction in tumor angiogenesis. Suppression of VEGF-induced angiogenesis: $\mathrm{BALB} / \mathrm{c}$ mice were vaccinated with empty vector, pLegumain, or pLegumain after either $\mathrm{CD} 8^{+}$or $\mathrm{CD} 4^{+} \mathrm{T}$ cell depletion in vivo. One week after the last vaccination, Matrigel was implanted s.c. into the midline of the abdomen of mice. Vascularization was induced by VEGF or bFGF. (A) The images were taken by a digital camera 6 days after Matrigel plug implantation. Additionally, the section of Matrigel plugs stained with Masson's trichrome indicate blood vessel growth in Matrigel plugs, as indicated by arrows. Magnification, $\times 50$. (B) Quantification of vessel growth was performed after in vivo staining of endothelium with FITC-labeled isolectin B4 and evaluation by fluorimetry. There was a decrease in the VEGF-induced neovasculature only after vaccination with the vector encoding legumain but not after vaccination with the empty vector or with pLegumain after depletion of CD8+ T cells. ${ }^{* *} P<0.005,{ }^{*} P<0.01$ compared with the legumain treatment group. (C) Immunohistochemical staining was performed and evaluated by confocal microscopy. The cross-sections of Matrigel plugs were stained to determine the cell type that grew in or migrated into these plugs. The images indicate that endothelial cells with the CD31 marker or macrophages with the CD68 marker grew in or migrated into Matrigel plugs as indicated (magnification, $\times 350)$. H\&E staining served as a control (magnification $\times 50)$.

defend the host against pathogenic infections, M2 cells attenuate acute inflammatory reactions, potently scavenge cellular debris, and secrete a variety of pro-growth and angiogenic factors essential for the repair of injured tissues. In addition, macrophages derived from healthy or inflamed tissue are capable of lysing tumor cells, expressing immunostimulatory cytokines, and presenting tumor-associated antigens to stimulate the proliferation and antitumor functions of T and NK cells. However, M2 macrophages, such as TAMs, show reduced levels of these activities. This may be the result of their exposure to tumor-derived antiinflammatory molecules such as IL-4, IL-10, TGF- $\beta 1$, and prostaglandin $\mathrm{E}_{2}(22,23)$. Indeed, this finding prompted Mantovani and col- legumain on the macrophage cell line RAW. This finding can best be understood when one considers that macrophages are derived from peripheral blood and differentiate into M2 macrophages once they are recruited into tumor sites where IL-4, IL-13, and IL-10 are released by tumor cells and tumor stromal cells $(3,19,29,31)$. Thus, targeting of $\mathrm{M} 2$ macrophages expressing legumain not only benefits suppression of tumor growth and metastases but also maintains the normal functions of macrophages with the M1 phenotype.

The relationship between infiltration by TAMs and prognosis in tumor patients has also been indicated by several studies $(1,5,32$, 33 ), which concluded that the greater the macrophage infiltration, the worse the prognosis. Several lines of evidence indicate that a 
symbiotic relationship exists in the tumor stroma between cancer cells and TAMs, whereby cancer cells attract TAMs and sustain their survival, while TAMs respond to tumor-derived molecules by producing important growth factors and extracellular matrix enzymes, which, in turn, stimulate tumor proliferation, angiogenesis, and invasion of surrounding tissues $(18,22,26,34)$. Thus, the attenuation of TAMs in the tumor environment can serve as an effective strategy to remodel the tumor stroma and to alter the tumor microenvironment (35).

In our study, a DNA construct encoding legumain evoked a robust CTL response against this asparaginyl endopeptidase, which functions as a stress protein that is highly overexpressed by TAMs. This immune response was shown to be MHC class I antigen restricted and $\mathrm{CD}^{+} \mathrm{T}$ cell specific. Importantly, our data also demonstrated that after immunization with the legumain-based DNA vaccines, the density of F4/80 $/ \mathrm{CD}^{2} 06^{+}$macrophages, i.e., TAMs, decreased dramatically. Furthermore, a variety of factors such as VEGF, MMP-9, and TGF- $\beta$ that are released by TAMs were shown to be at low levels in both the supernatant of cultured tumor cells and mouse serum. Thus, it is well known that VEGF and metalloproteinase MMP-9 play important roles during the formation of the tumor vasculature and initiation of tumor angiogenesis. TAMs are important in this regard, since they produce both VEGF and MMP-9 (36). Progressively intensifying angiogenesis is associated with the upregulated expression of VEGF (37) and extracellular proteases, such as MMP-9 $(8,36)$, whereas TGF- $\beta$ is known as an important growth factor involved in the migration of tumor cells toward blood vessels. In fact, TGF- $\beta$ can provide proliferative and antiapoptotic signals to tumor cells as well as activate urokinase-type plasminogen activators (uPAs), which might contribute to the extracellular matrix breakdown that is required for vascular invasion to occur (38). Significantly, our data demonstrate that once TAMs were abrogated in the tumor tissue by specific CD $8^{+}$CTLs, the tumor cells changed their character by becoming less malignant and less invasive. Also, the formation of a neovasculature in tumor tissues was reduced, since all of the factors released by TAMs that contribute to tumor angiogenesis were drastically reduced. Additionally, TAMs were reported to be involved in immune suppression and tolerance in the tumor microenvironment (39). It is also well known that TAMs may inhibit $\mathrm{T}$ cell responses by inducing apoptosis of activated T cells via upregulation of NO, PGs, TNF- $\alpha$ release, and arginase activity $(40,41)$. These may be some of the reasons for tumor immune tolerance, a notion also supported by our data. After abrogation of TAMs, the specific CD8 T cell activity was markedly upregulated, further supporting the contention that our anti-TAM approach could be a good strategy to break immune tolerance against tumors.

Furthermore, our hypothesis that a therapeutic approach using a legumain-based DNA vaccine to target TAMs holds much promise was strongly supported by data obtained in 3 tumor metastasis models used in our study. Thus, in the 4T1 spontaneous mouse breast carcinoma metastasis model, a significant increase in life span was obtained, as $75 \%$ (6/8) mice survived up to 3 months after 4T1 tumor cell inoculation into the mammary gland, once surgical resection of the primary tumor was followed by treatment with the legumain-based DNA vaccine. It was even more impressive that $62 \%(5 / 8)$ mice revealed no lung metastases at all. Similar results were obtained in prophylactic settings in the other 2 tumor models, i.e., D121 non-small cell lung carcinoma and CT26 colon carcinoma. These additional confirmatory data strengthen our contention that targeting of TAMs to remodel the tumor microenvironment might be a universal antitumor strategy for suppressing tumor cell invasion and metastases by reducing the concentration of factors released by TAMs that otherwise promote tumor growth and metastasis.

In summary, we critically evaluated the antitumor efficacy of targeting TAMs via the induction of a specific $\mathrm{CD}^{+} \mathrm{T}$ cell response against legumain, which we identified for the first time as being a highly overexpressed target molecule on TAMs. In these experiments, we demonstrated that abrogation of TAMs in tumor tissues effectively decreased the release of several pro-tumor growth and angiogenic factors. It is likely that such an antitumor strategy could be widely applicable and relevant for possible clinical applications.

\section{Methods}

Animals, bacterial strains, and cell lines. Female BALB/c and C57BL/ 6 mice, 6-8 weeks of age, were purchased from The Scripps Research Institute Rodent Breeding Facility. The double-attenuated S. typhimurium strain RE88 ( aro $^{-}$; dam $)^{-}$) was obtained from Remedyne Corp. The murine CT26 colon cancer cell line was kindly provided by I.J. Fidler (MD Anderson Cancer Center, Houston, Texas, USA), and the murine D121 non-small cell lung carcinoma cells were a gift from L. Eisenbach (Weizmann Institute of Science, Rehovot, Israel). The murine $4 \mathrm{~T} 1$ breast carcinoma cells were kindly provided by Suzanne Ostrand-Rosenberg (University of Maryland, College Park, Maryland, USA).

Immunohistochemical analyses. These were performed on 4T1 tumor tissues and Matrigel plug sections. Legumain expression of macrophages was identified on $4 \mathrm{~T} 1$ tumor tissue sections with biotinylated rat antimouse CD68 mAb (BD Biosciences - Pharmingen), with GFP-conjugated streptavidin being the secondary reporter reagent. Rabbit anti-legumain antiserum was prepared by immunization with purified human legumain produced in Escherichia coli. (10) The reaction was visualized with Texas red-conjugated streptavidin. Additionally, 4T1 tumor tissue sections and Matrigel plug sections were fixed and stained with MMP-9, VEGF, TGF- $\beta$, and F4/80 antibodies (eBioscience and Santa Cruz Biotechnology Inc.) in 4T1 tumor tissue section, while CD68 and CD31 Abs (BD Biosciences - Pharmingen) were used in Matrigel plug sections. All tissue sections were visualized with Texas red- or GFP-conjugated streptavidin as the secondary reporting reagent, and the slides were analyzed with laser scanning by confocal microscopy (Bio-Rad). All the images were captured by a SPOT Cooled Color Digital Camera System (Diagnostic Instruments Inc.).

Vector construction, protein expression, and transformation of S. typhimurium with DNA vaccine plasmids. Two constructs were made based on the vector pCMV (Invitrogen). The pLegumain construct consisted of polyubiquitinated, full-length murine legumain. The empty vector construct served as a control. Protein expression of legumain was demonstrated by Western blotting with a polyclonal rabbit anti-murine legumain $\mathrm{Ab}$ as well as anti-murine $\beta$-actin $\mathrm{Ab}$ (Santa Cruz Biotechnology Inc.) as a loading control. The specific protein was detected with a goat anti-rabbit HRP-conjugated IgG Ab (Bio-Rad). Attenuated S. typhimurium ( $\operatorname{aro} A^{-} ;$dam $\left.^{-}\right)$were transduced with DNA vaccine plasmids by electroporation as described in our previous publications $(42,43)$.

Immunization and tumor cell challenge. For the prophylactic model, BALB/c or C57BL/6 mice were each divided into 3 experimental groups $(n=8)$ and immunized with PBS, empty vector, or pLegumain. All mice were challenged by i.v. injection with $5 \times 10^{4} \mathrm{CT} 26$ cells $(\mathrm{BALB} / \mathrm{c})$ or $2 \times 10^{5} \mathrm{D} 121$ cells $(\mathrm{C} 57 \mathrm{BL} / 6)$ or injected in the mammary gland fat pad with $7 \times 10^{3}$ $4 \mathrm{~T} 1$ cells $(\mathrm{BALB} / \mathrm{c}), 1$ week after the last immunization, to induce either experimental or spontaneous pulmonary metastases. The lung weights 
in experimental or control groups were determined 24 days after tumor cell challenge. For the therapeutic model, BALB/c mice were divided into 3 experimental groups $(n=8)$ and first injected in the fat pad with $7 \times 10^{3}$ $4 \mathrm{~T} 1$ cells on day 0 and then immunized 3 times with DNA vaccine starting on days 3,7 , and 11 , and primary tumor was excised on day 12 . The experiment was terminated 24 days after primary tumor excision to determine lung weights and metastasis scores or mouse survival rates.

In vivo depletion of $C D 4^{+}$or $C D 8^{+} T$ cells, cytotoxicity, and ELISPOT assays. Analysis of the depletion of $\mathrm{CD}^{+}{ }^{+}$or $\mathrm{CD}^{+} \mathrm{T}$ cells in vivo was performed as previously described (44). Cytotoxicity was measured and calculated by a standard ${ }^{51} \mathrm{Cr}$ release assay as previously reported (45). ELISPOT assays were performed with an ELISPOT kit (BD Biosciences - Pharmingen) according to instructions provided by the manufacturer.

In vivo Matrigel angiogenesis assay. Matrigel was used for evaluating the suppression of angiogenesis after vaccination. Briefly, BALB/c mice were injected s.c. 2 weeks after the last vaccination, in the sternal region, with Growth Factor Reduced BD Matrigel (BD Biosciences) containing bFGF-2 (200 ng/plug) and 4T1 tumor cells $\left(5 \times 10^{3} /\right.$ plug $)$ that were previously irradiated with 1,000 Gy. The endothelium was stained 6 days after Matrigel implantation by i.v. injection with Bandeiraea simplicifolica lectin I (isolectin B4), conjugated with fluorescein (Vector Laboratories). This was done along with staining the endothelium of control animals, and 30 minutes later, mice were sacrificed, Matrigel plugs extracted, and fluorescence evaluated by fluorimetry. Additionally, the Matrigel plugs were removed 6 days after Matrigel implantation, fixed in Bouin's solution for 24 hours, and then embedded in paraffin. All tissues were sectioned, mounted onto slides, and stained with Masson's trichrome. All of the images were captured by a SPOT cooled color digital camera system (Diagnostic Instruments Inc.)

Flow cytometry. DC cell markers were determined by staining freshly isolated lymphocytes from successfully vaccinated mice and control mice with PElabeled anti-CD11c Ab in combination with FITC-conjugated anti-CD40, anti-CD80 Ab, and Abs against MHC class II antigen. Macrophages bearing high levels of CD206 ${ }^{+}$and $\mathrm{F} 4 / 80^{+}$were quantified by 2 -color flow analysis. Tumor cells were isolated from successfully vaccinated $\mathrm{BALB} / \mathrm{c}$ mice and then stained with anti-CD206 Ab conjugated with PE (Cell Sciences),
anti-F4/80 Ab conjugated with APC, and anti-legumain Ab conjugated with FITC, followed by FACS analyses. All antibodies were purchased from BD Biosciences - Pharmingen. IFN- $\gamma$ release at the intracellular level was determined in lymphocytes of Peyer's patches obtained 3 days after onetime immunization and stained with APC-conjugated anti-CD8 Ab. Cell were fixed, permeabilized, and subsequently stained with PE-labeled antiIFN- $\gamma \mathrm{Ab}$ to detect intracellular expression of IFN- $\gamma$.

Migration assay. Cell migration assays were performed using modified Boyden chambers (Transwell; Corning Inc.). Transwell migration assays were performed with tumor cells harvested from tumor tissue of either vaccine-treated or control groups of mice. After 4 hours culture, the cells on the lower surface of wells were fixed with $1 \%$ paraformaldehyde, stained with $1 \%$ crystal violet, and counted (46).

Statistics. The statistical significance of differential findings between experimental groups and controls was determined by Student's $t$ test. Findings were regarding as significant if 2-tailed $P$ values were less than 0.05 . Kaplan-Meier analysis was used to evaluate the survival of mice.

\section{Acknowledgments}

We thank K. Cairns for editorial assistance. This work was supported by grants DAMD17-02-0137 and DAMD17-02-0562 (to R. Xiang) from the US Department of Defense; California TobaccoRelated Disease Research Program grant 12 RT-0002 (to R.A. Reisfeld); grant SFP 1330 from the EMD Lexigen Research Center (to R.A. Reisfeld); as well as Congressionally Directed Medical Research Program grants W81XWH-05-1-0091 and W81XWH-051-0318 (to C. Liu). This is The Scripps Research Institute's manuscript number 17696-IMM.

Received for publication December 12, 2005, and accepted in revised form May 23, 2006.

Address correspondence to: Rong Xiang, The Scripps Research Institute, R218, IMM13, 10550 North Torrey Pines Road, La Jolla, California 92037, USA. Phone: (858) 784-8124; Fax: (858) 784-2708; E-mail: rxiang@scripps.edu.
1. Oosterling, S.J., et al. 2005. Macrophages direct tumour histology and clinical outcome in a colon cancer model. J. Pathol. 207:147-155.

2. Emens, L.A., Reilly, R.T., and Jaffee, E.M. 2005 Breast cancer vaccines: maximizing cancer treatment by tapping into host immunity. Endocr. Relat. Cancer. 12:1-17.

3. Mills, C.D., Kincaid, K., Alt, J.M., Heilman, M.J., and Hill, A.M. 2000. M-1/M-2 macrophages and the Th1/Th2 paradigm. J. Immunol. 164:6166-6173.

4. Mantovani, A., et al. 2004. Infiltration of tumours by macrophages and dendritic cells: tumour-associated macrophages as a paradigm for polarized M2 mononuclear phagocytes. Novartis Found. Symp. 256:137-145.

5. Lewis, C., and Murdoch, C. 2005. Macrophage responses to hypoxia: implications for tumor progression and anti-cancer therapies. Am. J. Pathol. 167:627-635.

6. Mantovani, A., Allavena, P., and Sica, A. 2004. Tumour-associated macrophages as a prototypic type II polarised phagocyte population: role in tumour progression. Eur. J. Cancer. 40:1660-1667.

7. Allavena, P., et al. 2005. Anti-inflammatory properties of the novel antitumor agent yondelis (trabectedin): inhibition of macrophage differentiation and cytokine production. Cancer Res. 65:2964-2971.

8. Giraudo, E., Inoue, M., and Hanahan, D. 2004. An amino-bisphosphonate targets MMP-9-expressing macrophages and angiogenesis to impair cer- vical carcinogenesis. J. Clin. Invest. 114:623-633. doi:10.1172/JCI200422087.

9. Robinson, S.C., et al. 2003. A chemokine receptor antagonist inhibits experimental breast tumor growth. Cancer Res. 63:8360-8365.

10. Ishii, S. 1994. Legumain: asparaginyl endopeptidase. Methods Enzymol. 244:604-615.

11. Kembhavi, A.A., Buttle, D.J., Knight, C.G., and Barrett, A.J. 1993. The two cysteine endopeptidases of legume seeds: purification and characterization by use of specific fluorometric assays. Arch. Biochem. Biophys. 303:208-213.

12. Schlereth, A., Becker, C., Horstmann, C., Tiedemann, J., and Muntz, K. 2000. Comparison of globulin mobilization and cysteine proteinases in embryonic axes and cotyledons during germination and seedling growth of vetch (Vicia sativa L.). J. Exp. Bot. 51:1423-1433.

13. Chen, J.M., et al. 1997. Cloning, isolation, and characterization of mammalian legumain, an asparaginyl endopeptidase. J. Biol. Chem. 272:8090-8098.

14. Liu, C., Sun, C., Huang, H., Janda, K., and Edgington, T. 2003. Overexpression of legumain in tumors is significant for invasion/metastasis and a candidate enzymatic target for prodrug therapy. Cancer Res. 63:2957-2964.

15. Murthy, R.V., Arbman, G., Gao, J., Roodman, G.D. and Sun, X.F. 2005. Legumain expression in relation to clinicopathologic and biological variables in colorectal cancer. Clin. Cancer Res. 11:2293-2299.
16. Balkwill, F., Charles, K.A., and Mantovani, A. 2005. Smoldering and polarized inflammation in the initiation and promotion of malignant disease. Cancer Cell. 7:211-217.

17. Silzle, T., et al. 2003. Tumor-associated fibroblasts recruit blood monocytes into tumor tissue. Eur. J. Immunol. 33:1311-1320.

18. Pollard, J.W. 2004. Tumour-educated macrophages promote tumour progression and metastasis. Nat. Rev. Cancer. 4:71-78.

19. Ibe, S., Qin, Z., Schuler, T., Preiss, S., and Blankenstein, T. 2001. Tumor rejection by disturbing tumor stroma cell interactions. J. Exp. Med. 194:1549-1559.

20. Karin, M. 2005. Inflammation and cancer: the long reach of Ras. Nat. Med. 11:20-21.

21. Coussens, L.M., and Werb, Z. 2002. Inflammation and cancer. Nature. 420:860-867.

22. Bingle, L., Brown, N.J., and Lewis, C.E. 2002. The role of tumour-associated macrophages in tumour progression: implications for new anticancer therapies. J. Pathol. 196:254-265.

23. Leek, R.D., and Harris, A.L. 2002. Tumor-associated macrophages in breast cancer. J. Mammary Gland Biol. Neoplasia. 7:177-189.

24. Mantovani, A., Sozzani, S., Locati, M., Allavena, P., and Sica, A. 2002. Macrophage polarization: tumor-associated macrophages as a paradigm for polarized M2 mononuclear phagocytes. Trends Immunol. 23:549-555. 
25. Sica, A., et al. 2000. Autocrine production of IL-10 mediates defective IL-12 production and NF-kappa $B$ activation in tumor-associated macrophages. J. Immunol. 164:762-767.

26. Wyckoff, J., et al. 2004. A paracrine loop between tumor cells and macrophages is required for tumor cell migration in mammary tumors. Cancer Res. 64:7022-7029.

27. Sinha, P., Clements, V.K., and Ostrand-Rosenberg, S. 2005. Reduction of myeloid-derived suppressor cells and induction of M1 macrophages facilitate the rejection of established metastatic disease. J. Immunol. 174:636-645.

28. Misson, P., van den Brule, S., Barbarin, V., Lison, D., and Huaux, F. 2004. Markers of macrophage differentiation in experimental silicosis. J. Leukoc. Biol. 76:926-932.

29. Stein, M., Keshav, S., Harris, N., and Gordon, S. 1992. Interleukin 4 potently enhances murine macrophage mannose receptor activity: a marker of alternative immunologic macrophage activation. J. Exp. Med. 176:287-292.

30. Porcheray, F., et al. 2005. Macrophage activation switching: an asset for the resolution of inflammation. Clin. Exp. Immunol. 142:481-489.

31. Kuroda, E., and Yamashita, U. 2003. Mechanisms of enhanced macrophage-mediated prostaglandin $\mathrm{E} 2$ production and its suppressive role in Th1 acti- vation in Th2-dominant BALB/c mice. J. Immunol. 170:757-764

32. Barbera-Guillem, E., Nyhus, J.K., Wolford, C.C., Friece, C.R., and Sampsel, J.W. 2002. Vascular endothelial growth factor secretion by tumor-infiltrating macrophages essentially supports tumor angiogenesis, and IgG immune complexes potentiate the process. Cancer Res. 62:7042-7049.

33. Shimura, S., et al. 2000. Reduced infiltration of tumor-associated macrophages in human prostate cancer: association with cancer progression. Cancer Res. 60:5857-5861.

34. Knowles, H., Leek, R., and Harris, A.L. 2004. Macrophage infiltration and angiogenesis in human malignancy. Novartis Found. Symp. 256:189-200.

35. Lin, E.Y., and Pollard, J.W. 2004. Macrophages: modulators of breast cancer progression. Novartis Found. Symp. 256:158-168.

36. Hiratsuka, S., et al. 2002. MMP9 induction by vascular endothelial growth factor receptor- 1 is involved in lung-specific metastasis. Cancer Cell. 2:289-300.

37. Van Kempen, L.C., and Coussens, L.M. 2002. MMP9 potentiates pulmonary metastasis formation. Cancer Cell. 2:251-252.

38. De Wever, O., and Mareel, M. 2003. Role of tissue stroma in cancer cell invasion. J. Pathol. 200:429-447.

39. Condeelis, J., and Pollard, J.W. 2006. Macrophages: obligate partners for tumor cell migration, invasion, and metastasis. Cell. 124:263-266.

40. Saio, M., Radoja, S., Marino, M., and Frey, A.B. 2001. Tumor-infiltrating macrophages induce apoptosis in activated CD8(+) T cells by a mechanism requiring cell contact and mediated by both the cell-associated form of TNF and nitric oxide. J. Immunol. 167:5583-5593.

41. Kusmartsev, S., and Gabrilovich, D.I. 2005. STAT1 signaling regulates tumor-associated macrophagemediated T cell deletion. J. Immunol. 174:4880-4891.

42. Luo, Y., et al. 2003. Transcription factor Fos-related antigen 1 is an effective target for a breast cancer vaccine. Proc. Natl. Acad. Sci. U. S. A. 100:8850-8855.

43. Xiang, R., et al. 2000. An autologous oral DNA vaccine protects against murine melanoma. Proc. Natl. Acad. Sci. U. S. A. 97:5492-5497.

44. Ceredig, R., Lowenthal, J.W., Nabholz, M., and MacDonald, H.R. 1985. Expression of interleukin-2 receptors as a differentiation marker on intrathymic stem cells. Nature. 314:98-100.

45. Zhou, H., et al. 2005. T cell-mediated suppression of angiogenesis results in tumor protective immunity. Blood. 106:2026-2032.

46. Shi, X., Gangadharan, B., Brass, L.F., Ruf, W., and Mueller, B.M. 2004. Protease-activated receptors (PAR1 and PAR2) contribute to tumor cell motility and metastasis. Mol. Cancer Res. 2:395-402. 